\title{
EFEITO DA INTENSIDADE NO EXERCÍCIO ANTAGONISTA SOBRE O DESEMPENHO MUSCULAR NO MÉTODO AGONISTA/ANTAGONISTA: UMA REVISÃO NARRATIVA
}

${ }^{1}$ Eduardo Ramon Canuto da Luz ${ }^{2}$ Juliana Daniele de Araújo Silva ${ }^{3}$ Diógenes Candido Mendes Maranhão ${ }^{4}$ André Luiz Torres Pirauá

\section{RESUMO}

Introdução: O método agonista-antagonista (MAA) consiste na execução de dois exercícios para o mesmo segmento corporal, de forma combinada, sendo o primeiro deles à musculatura antagonista, visando a diminuição da co-contração. Objetivo: O objetivo do presente estudo foi verificar por meio de uma revisão narrativa, os efeitos da intensidade aplicada ao exercício antagonista, sobre o desempenho muscular do exercício agonista no MAA. Método: A pesquisa foi conduzida nas bases de dados eletrônicos Google Acadêmico, Lilacs, Scieloe Medline/Pubmed. Resultados e Discussão: Foram achados um total de 15 artigos, onde verificou-se que o método agonista antagonista parece ser eficaz para o aumento do desempenho e ativação muscular, e do volume total de trabalho, independente da intensidade aplicada, sendo essas as principais variáveis estudadas. Conclusão: Não é consensual qual a intensidade mais adequada deve ser aplicada no método, para se obter seus resultados, sendo assim uma lacuna para possíveis estudos futuros.

Palavras-Chave: Treinamento de Resistência, Força Muscular, Desempenho Atlético.

\section{EFFECT OF INTENSITY IN ANTAGONIST EXERCISE ON MUSCLE PERFORMANCE IN THE AGONIST/ANTAGONIST METHOD: A NARRATIVE REVIEW}

\begin{abstract}
Introduction: The agonist-antagonist (AAM) method consists of performing two exercises for the same body segment, in combination, the first one being the antagonist muscles, aiming to reduce cocontraction. Objective: The aim of the present study was to verify, through a narrative review, the effects of the intensity applied to the antagonist exercise, on the muscular performance of the agonist exercise in the MAA. Method: The search was conducted in the Google Scholar, Lilacs, Scieloe Medline/Pubmed electronic databases. Results and Discussion: A total of 15 articles were found, where it was found that the antagonist agonist method seems to be effective in increasing muscle performance and activation, and the total workload, regardless of the intensity applied, these being the main variables studied. Conclusion: There is no consensus on which intensity should be applied in the method, in order to obtain its results, which is a gap for possible future studies.
\end{abstract}

Keywords: Endurance Training, Muscle Strength, Athletic Performance.

${ }^{1}$ Centro Universitário Tabosa de Almeida, eduardo.ramon.12@hotmail.com. Rua Duque de Caxias, 232, Centro. CEP: 55004-300 - Caruaru, Pernambuco.

${ }^{2}$ Universidade Federal de Pernambuco

${ }^{34}$ Universidade Federal Rural de Pernambuco

E-mails: eduardo.ramon.12@hotmail.com; julianadanielearaujo@gmail.com; diogesmendes@gmail.com; andre.piraua@ufrpe.br. 
O método agonista-antagonista (MAA) consiste na execução de dois exercícios para o mesmo segmento corporal, de forma combinada ou pareada, sendo o primeiro deles antagonista ao grupamento muscular prioritário (agonista) (PAZ et al., 2014). O objetivo desse método é gerar uma ativação prévia da musculatura antagonista, afim de reduzir os níveis de co-contração, para favorecer o desempenho do agonista (MAIA et al., 2014; PAZ et al., 2017).

Dentre as possibilidades de aplicação, observa-se que MAA pode ser executado de forma combinada, quando os exercícios são realizados sem intervalos de recuperação, ou pareada, com intervalos que podem variar de um a cinco minutos do primeiro para o segundo exercício (PAZ et al., 2015). Além disso, o estímulo da musculatura antagonista pode ser administrado por meio de um exercício do Treinamento de Força (MAIA et al., 2014; PAZ et al., 2014) ou por um exercício de alongamento (MIRANDA et al., 2015; PAZ et al., 2012). De modo geral, observa-se que menores intervalos de

\section{MÉTODO}

Trata-se de uma revisão narrativa que objetivou verificar os efeitos da intensidade aplicada ao exercício antagonista, sobre o desempenho muscular do exercício agonista no MAA. A revisão foi conduzida no período de setembro de 2017 a agosto de 2021. Para a análise da qualidade dos estudos, foi utilizado a escala TESTEX (SMART et al., 2015).

A pesquisa foi conduzida nas bases de dados eletrônicos Google Acadêmico, Literatura Latino-Americana e do Caribe em Ciências da Saúde (LILACS), Scientific Electronic Library Online (SCIELO) e National Library of Medicine National Institutes of Health (PUBMED). Os descritores foram selecionados de acordo com os resultados obtidos em consulta ao Descritores em Ciências da Saúde (DeCS), onde obteve-se: Treinamento de Resistência, Eletromiografia; e ao Medical Subject Headings (MeSH), onde obteve-se: "Strength training", "Resistance Training", "Electromyography". Foram utilizadas palavras-chaves para facilitar o filtro da pesquisa, são elas: Treinamento de força, coativação, performance muscular, ativação muscular, séries pareadas. Igualmente, as palavras-chaves utilizadas em língua inglesa recuperação entre os exercícios no MAA promovem maiores níveis de atividade eletromiográfica para a musculatura agonista do movimento (MAIA et al., 2014) e também um aumento sobre o número de repetições executadas, repercutindo sobre o volume total do treinamento (PAZ et al., 2016; PAZ et al., 2014; ROBBINS et al., 2010).

Ao revisar a literatura, observa-se que não há um consenso sobre a intensidade adotada para o exercício antagonista, que variaram de moderada (PAZ et al., 2014) a alta (ROBBINS et al., 2010). Assim, não se sabe quais as possíveis implicações da intensidade sobre a redução da co-contração, bem como os efeitos sobre o desempenho muscular dos grupamentos agonistas do movimento durante a realização do MAA. Portanto, o objetivo do presente estudo foi verificar, por meio de uma revisão narrativa, os efeitos da intensidade aplicada ao exercício antagonista, sobre o desempenho muscular (atividade eletromiográfica, número de repetições e volume total de trabalho) do exercício agonista no

MAA.

foram: "Coactivation", "strength performance", "muscle activation" e "paired set".

Adicionalmente, foram utilizados os operadores booleanos "AND" e "OR" para as bases na língua inglesa e seus correspondentes "E" e "OU" para as bases de língua portuguesa. Dessa forma, a partir da seleção dos descritores e palavras-chave foi criada, a seguinte expressão de busca: "Strength training" OR "Resistance Training" AND Electromyography OR Coactivation OR Strength Performance OR Muscle Activation AND Paired Sef', e o seu correspondente em português: "Treinamento de Força OU Treinamento Resistido E Eletromiografia OU Coativação OU Performance Muscular OU Ativação Muscular OU Séries Pareadas".

Os processos de busca, seleção e avaliação dos artigos foram divididos da seguinte forma: um pesquisador foi responsável pela busca, seleção e avaliação inicial. Enquanto isso, outros dois foram responsáveis pela busca complementar e atualização dos estudos. Para possíveis divergências encontradas entre a busca, seleção, avaliação e atualização dos estudos, um terceiro pesquisador foi acionado, afim de 
revisar a literatura e emitir um parecer final. $O$ procedimento de localização e seleção dos artigos científicos ocorreu em dois estágios. No primeiro estágio, os artigos foram selecionados a partir da leitura dos seus títulos e resumos. No segundo estágio, foram acessados e avaliados os textos completos, dos artigos publicados até o mês de agosto do ano de 2021, que abordam os efeitos do método agonista-antagonista sobre a atividade muscular ou o número de repetições realizadas.

Foram considerados apenas artigos originais (agudos ou crônicos), publicados em

\section{RESULTADOS}

No quadro 1 são apresentadas as características metodológicas dos 15 estudos selecionados, bem como as características das intervenções realizadas, exercícios e resultados encontrados. Também são apresentados os valores obtidos da escala TESTEX utilizada para avaliar os ensaios clínicos randomizados, bem como as frequências de pontuação obtidas em cada critério avaliado pelo TESTEX, subdividido em qualidade do estudo (pontuação máxima de 5 pontos) e qualidade do relatório (pontuação periódicos na língua inglesa e portuguesa, conduzidos com seres humanos, de ambos os sexos, ativos ou inativos fisicamente, sem limites para a faixa etária, nível de treinamento (iniciantes, intermediários ou avançados) e que avaliaram como desfechos a atividade eletromiográfica, número de repetições realizados e volume total de trabalho. Foram excluídos artigos duplicados, de revisão, teses ou que não especificaram a realização do método com o pressuposto da redução da coativação muscular, visando a melhoria do desempenho muscular da musculatura agonista. máxima de 10 pontos), totalizando os 15 pontos.

Quadro 1 - Resultados da sumarização dos estudos que avaliaram a aplicação do MAA no treinamento de força.

\begin{tabular}{|c|c|c|c|c|c|}
\hline Autor & Amostra & $\begin{array}{l}\text { Variáveis } \\
\text { principais }\end{array}$ & Protocolo & Resultados & Qualidade \\
\hline $\begin{array}{l}\text { COSTA et al., } \\
(2020) .\end{array}$ & $\begin{array}{c}20 \\
\text { homens } \\
\text { 76,9 anos } \\
\text { Treinados }\end{array}$ & $\begin{array}{l}\text { Número de } \\
\text { repetições e } \\
\text { volume de } \\
\text { carga }\end{array}$ & $\begin{array}{l}\text { Agonista: Flexor } \\
\text { de joelho } \\
\text { Antagonista: } \\
\text { Extensor de joelho } \\
\text { Exercícios: Flexão } \\
\text { e extensão de } \\
\text { joelho } \\
\text { Séries: } 1 \text { série de } 8 \\
\text { repetições ou } 1 \\
\text { série de } 12 \\
\text { repetições } \\
\text { Intervalo: Não } \\
\text { informado }\end{array}$ & $\begin{array}{l}\text { Manutenção } \\
\text { do número } \\
\text { de repetições } \\
\text { e volume de } \\
\text { carga }\end{array}$ & $9 / 15$ \\
\hline
\end{tabular}


Exercício físico e desempenho muscular

\begin{tabular}{|c|c|c|c|c|c|}
\hline & & & $\begin{array}{c}\text { Intensidade: } 75 \% \\
\text { de } 1 \text { RM no } \\
\text { antagonista ou } 50 \% \\
\text { de } 1 \text { RM no } \\
\text { Antagonista, até à } \\
\text { falha concêntrica }\end{array}$ & & \\
\hline $\begin{array}{c}\text { PAZ; MAIA } \\
(2016) .\end{array}$ & $\begin{array}{c}15 \\
\text { homens } \\
22 \text { anos } \\
\text { Treinados }\end{array}$ & $\begin{array}{l}\text { Atividade } \\
\text { eletromiográfica; } \\
\text { Volume } \\
\text { total de treino }\end{array}$ & $\begin{array}{c}\text { Agonista: Peitoral } \\
\text { Antagonista: Dorsal } \\
\text { Exercícios: Supino } \\
\text { Vertical; Remada } \\
\text { Aberta/Supino } \\
\text { vertical + Remada } \\
\text { Aberta } \\
\text { Séries: } 3 \\
\text { Intervalo: 2min } \\
\text { Intervalo/Exercícios } \\
\text { : } 2 \text { minutos } \\
\text { Intensidade: } \\
\text { Agonista: 10RM; } \\
\text { Antagonista: } \\
\text { 10RM }\end{array}$ & $\begin{array}{l}\uparrow \text { Volume de } \\
\text { treinamento }\end{array}$ & $7 / 15$ \\
\hline $\begin{array}{l}\text { MIRANDA et } \\
\text { al., (2015). }\end{array}$ & $\begin{array}{c}10 \\
\text { homens } \\
22 \text { anos } \\
\text { Treinados }\end{array}$ & $\begin{array}{l}\text { Número de } \\
\text { repetições; } \\
\text { Atividade } \\
\text { eletromiográfica }\end{array}$ & $\begin{array}{l}\text { Agonista: Dorsal/ } \\
\text { Antagonista: } \\
\text { Peitoral } \\
\text { Exercícios: } \\
\text { Remada } \\
\text { aberta/Alongament } \\
\text { o estático } \\
\text { Séries: } 3 \text { séries } \\
\text { (repetições } \\
\text { máximas) } \\
\text { Intervalo: } 2 \\
\text { minutos } \\
\text { Intervalo/Exercíci } \\
\text { os: } 1 \text { '20" } \\
\text { Intensidade: }\end{array}$ & $\begin{array}{l}\uparrow \text { Número de } \\
\text { repetições }\end{array}$ & $8 / 15$ \\
\hline
\end{tabular}


Exercício físico e desempenho muscular

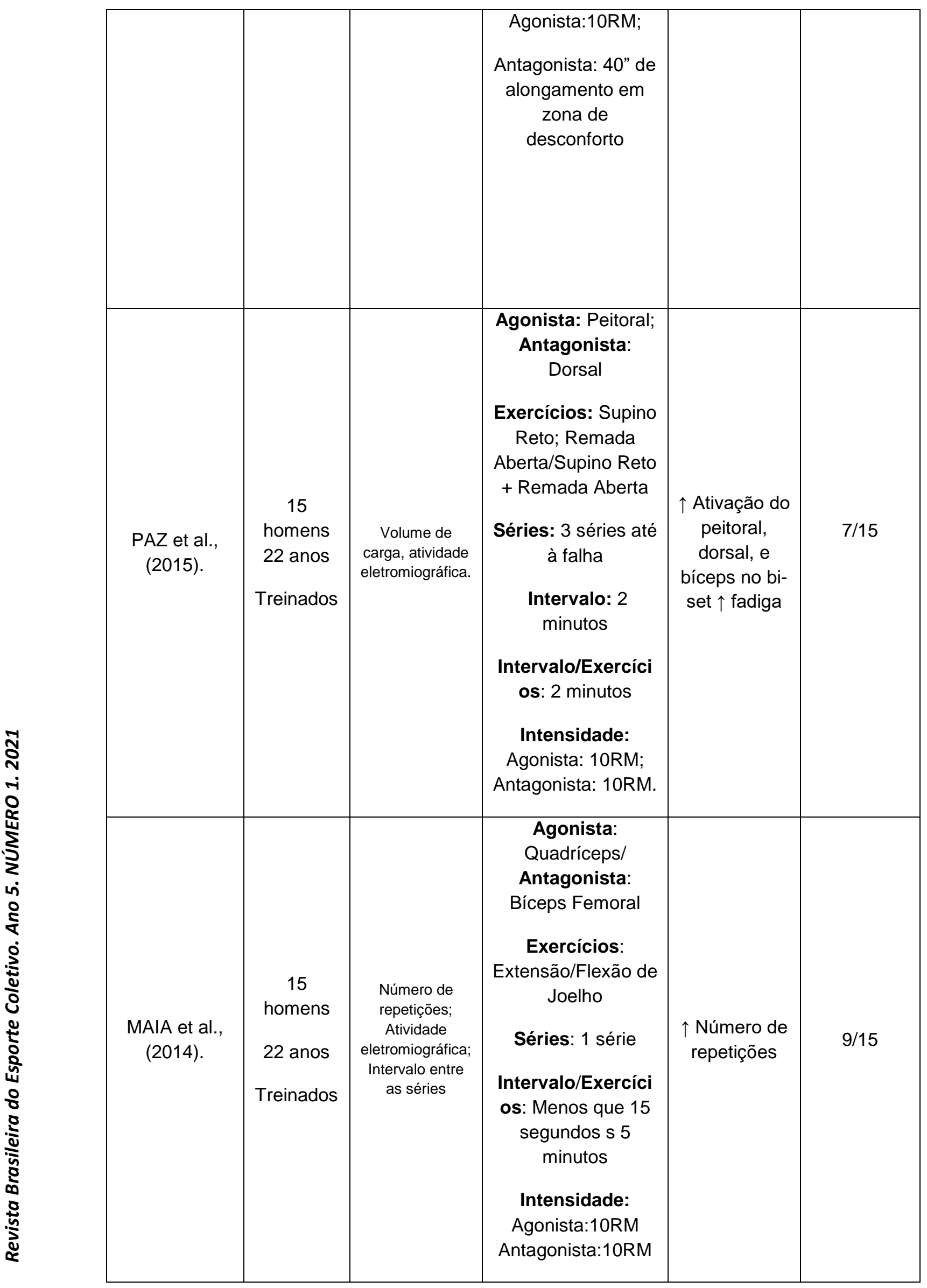


Exercício físico e desempenho muscular

\begin{tabular}{|c|c|c|c|c|c|}
\hline PAZ (2014) & $\begin{array}{c}10 \\
\text { homens } \\
22 \text { anos } \\
\text { Treinados }\end{array}$ & $\begin{array}{l}\text { Número de } \\
\text { repetições; } \\
\text { Atividade } \\
\text { Eletromiográfica }\end{array}$ & $\begin{array}{l}\text { Agonista: Bíceps } \\
\text { Femoral/ } \\
\text { Antagonista: } \\
\text { Quadríceps } \\
\text { Exercícios: Mesa } \\
\text { flexora/Alongament } \\
\text { o Estático } \\
\text { Séries: } 1 \\
\text { Intervalo/Exercíci } \\
\text { os: Imediato }\end{array}$ & $\begin{array}{l}\uparrow \text { Volume } \\
\text { total de } \\
\text { treinamento }\end{array}$ & $6 / 15$ \\
\hline $\begin{array}{l}\text { STOCK; } \\
\text { THOMPSON } \\
\text { (2014). }\end{array}$ & $\begin{array}{c}54 \\
\text { participan } \\
\text { tes } \\
23 \text { anos } \\
\text { Não } \\
\text { treinados } \\
\text { Grupo } 1 \\
17 \\
\text { homens e } \\
17 \\
\text { mulheres } \\
\text { Grupo } \\
\text { Controle } \\
9 \text { homens } \\
\text { e } 12 \\
\text { mulheres }\end{array}$ & $\begin{array}{c}\text { Pico de } \\
\text { torque; } \\
\text { Coativação }\end{array}$ & $\begin{array}{c}\text { Agonista: } \\
\text { Quadríceps } \\
\text { Antagonista: } \\
\text { Bíceps Femoral } \\
\text { Exercícios: } \\
\text { Levantamento } \\
\text { Terra } \\
\text { Séries: } 5 \\
\text { Intervalo: } 3 \\
\text { minutos } \\
\text { Intensidade: } \\
\text { Agonista } \\
\text { (8RM) }\end{array}$ & $\begin{array}{c}\uparrow \text { Pico de } \\
\text { torque }\end{array}$ & $5 / 15$ \\
\hline $\begin{array}{l}\text { MAEO et al., } \\
(2014) .\end{array}$ & $\begin{array}{c}60 \\
\text { homens } \\
21,4 \text { anos } \\
\text { Experiênc } \\
\text { ia em } \\
\text { treinamen } \\
\text { to não } \\
\text { informada }\end{array}$ & $\begin{array}{c}\text { Adaptações } \\
\text { Neuromuscula } \\
\text { res }\end{array}$ & $\begin{array}{l}\text { Agonista: Bíceps } \\
\text { Antagonista: } \\
\text { Tríceps } \\
\text { Exercícios: } \\
\text { Contração a 90ำ } \\
\text { cotovelo } \\
\text { Séries: } 5 \times 10 \\
\text { Intensidade: } \\
\text { Agonista: 4"+4" } \\
\text { relaxamento }\end{array}$ & $\begin{array}{c}\uparrow \text { Torque } \uparrow \\
\text { Ativação } \\
\text { Agonista. } \\
\text { Sem } \\
\text { diferença } \\
\text { antagonista }\end{array}$ & $6 / 15$ \\
\hline
\end{tabular}


Exercício físico e desempenho muscular

\begin{tabular}{|c|c|c|c|c|c|}
\hline $\begin{array}{l}\text { MIRANDA et } \\
\text { al., (2014). }\end{array}$ & $\begin{array}{c}11 \\
\text { homens } \\
24 \text { Anos } \\
\text { Treinados }\end{array}$ & $\begin{array}{l}\text { Desempenho } \\
\text { de repetições }\end{array}$ & $\begin{array}{c}\text { Agonista: Bíceps } \\
\text { braquial/ Bíceps } \\
\text { femoral } \\
\text { Antagonista: } \\
\text { Tríceps braquial; } \\
\text { Quadríceps } \\
\text { Exercícios: Rosca } \\
\text { Scott; Mesa flexora } \\
\text { Séries: } 1 \\
\text { Intervalo: Não } \\
\text { houve } \\
\text { Intensidade: } 90 \% \\
\text { de } 10 \text { RM }\end{array}$ & $\begin{array}{l}\uparrow \text { Repetições } \\
\text { máximas }\end{array}$ & $7 / 15$ \\
\hline $\begin{array}{c}\text { CUNHA, } \\
\text { CARREGAR } \\
\text { O, } \\
\text { MARTORELL } \\
\text { I (2013). }\end{array}$ & $\begin{array}{c}33 \\
\text { homens } \\
21 \text { anos } \\
\text { Sem } \\
\text { experiênci } \\
\text { a com } \\
\text { treinamen } \\
\text { to } \\
\text { resistido }\end{array}$ & $\begin{array}{c}\text { Respostas } \\
\text { Neuromuscu } \\
\text { lares }\end{array}$ & $\begin{array}{c}\text { Agonista: } \\
\text { Quadríceps } \\
\text { Antagonista: } \\
\text { Bíceps Femoral } \\
\text { Exercícios: Ext. } \\
\text { Joelho/Extensão + } \\
\text { Flexão Joelho } \\
\text { (isocinético) } \\
\text { Séries: } 4 \times 10 \\
\text { repetições } \\
\text { Intervalo: } 1 \text { minuto } \\
\text { Intensidade: } \\
\text { Agonista: Não } \\
\text { informado } \\
\text { Antagonista: Não } \\
\text { informado }\end{array}$ & $\begin{array}{c}\downarrow \\
\text { Classificação } \\
\text { de } \\
\text { desenvolvim } \\
\text { ento de } \\
\text { aceleração e } \\
\text { do tempo } \\
\text { para pico de } \\
\text { torque }\end{array}$ & $10 / 15$ \\
\hline $\begin{array}{c}\text { PAZ et al., } \\
\text { (2012). }\end{array}$ & $\begin{array}{c}10 \\
\text { homens } \\
22 \text { anos } \\
\text { Treinados }\end{array}$ & $\begin{array}{l}\text { Ativação do } \\
\text { Agonista, } \\
\text { Número de } \\
\text { repetições }\end{array}$ & $\begin{array}{l}\text { Agonista: Dorsal } \\
\text { Antagonista: } \\
\text { Peitoral } \\
\text { Exercícios: } \\
\text { Remada } \\
\text { sentada/Remada } \\
\text { Sentada + } \\
\text { Propriocepção } \\
\text { Séries: } 1 \text { série de } \\
6 \text { segundos de } \\
\text { contração e depois } \\
4 \text { segundos de } \\
\text { alongamento }\end{array}$ & $\begin{array}{l}\uparrow \text { Número de } \\
\text { repetições }\end{array}$ & $8 / 15$ \\
\hline
\end{tabular}


Exercício físico e desempenho muscular

\begin{tabular}{|c|c|c|c|c|c|}
\hline & & & $\begin{array}{c}\text { Intensidade: } \\
\text { Agonista: } 10 \text { cargas } \\
\text { de repetições } \\
\text { máximas; } \\
\text { Antagonista: } \\
\text { Propriocepção }\end{array}$ & & \\
\hline $\begin{array}{l}\text { NOBRE, } \\
\text { FIGUEIREDO } \\
(2010) .\end{array}$ & $\begin{array}{c}8 \text { homens } \\
24 \text { Anos } \\
\text { Treinados }\end{array}$ & $\begin{array}{l}\text { Número de } \\
\text { repetições }\end{array}$ & $\begin{array}{c}\text { Agonista: } \\
\text { Quadríceps/Antag } \\
\text { onista: Bíceps } \\
\text { Femoral; } \\
\text { Exercícios: } \\
\text { Cadeira } \\
\text { Extensora/Mesa } \\
\text { Flexora; } \\
\text { Séries: 1 série } \\
\text { Intervalo: sem } \\
\text { intervalo; } \\
\text { Intervalo/Exercíci } \\
\text { os: Menos que 15 } \\
\text { segundos } \\
\text { Intensidade: } \\
\text { Agonista:10RM; } \\
\text { Antagonista:10RM }\end{array}$ & $\begin{array}{l}\uparrow \text { Número de } \\
\text { repetições }\end{array}$ & $10 / 15$ \\
\hline $\begin{array}{l}\text { ROBBINS et } \\
\text { al., (2010). }\end{array}$ & $\begin{array}{c} \\
18 \\
\text { homens } \\
24,6 \text { anos } \\
\text { Treinados }\end{array}$ & $\begin{array}{l}\text { Volume de } \\
\text { carga; saída de } \\
\text { energia; } \\
\text { atividade } \\
\text { eletromiográfica } \\
\text { e eficiência }\end{array}$ & $\begin{array}{l}\text { Agosnista: Peitoral } \\
\text { maior, deltoide, } \\
\text { latíssimo do dorso } \\
\text { e trapézio } \\
\text { Antagonista: } \\
\text { Peitoral maior, } \\
\text { deltoide, latíssimo } \\
\text { do dorso e trapézio } \\
\text { Exercícios: } \\
\text { Arremesso de } \\
\text { supino } \\
\text { Séries: } 4 \\
\text { Intervalo: } 2 \\
\text { minutos }\end{array}$ & $\begin{array}{c}\uparrow \text { Eficiência } \\
\text { nas variáveis } \\
\text { analisadas } \\
\text { com o } \\
\text { treinamento } \\
\text { complexo }\end{array}$ & $6 / 15$ \\
\hline
\end{tabular}


Exercício físico e desempenho muscular

\begin{tabular}{|c|c|c|c|c|c|}
\hline & & & $\begin{array}{c}\text { Intensidade: } 30 \text { a } \\
60 \% \text { de } 1 \mathrm{RM}\end{array}$ & & \\
\hline $\begin{array}{l}\text { ROBBINS et } \\
\text { al., (2009). }\end{array}$ & $\begin{array}{c}50 \\
\text { homens } \\
\text { Idade não } \\
\text { informada } \\
\text { Treinados }\end{array}$ & $\begin{array}{l}\text { 1RM, Potência, } \\
\text { Atividade } \\
\text { eletromiográfica }\end{array}$ & $\begin{array}{l}\text { Agonista: Peitoral } \\
\text { Antagonista: } \\
\text { Dorsal } \\
\text { Exercícios: Supino } \\
\text { Reto/Remada } \\
\text { Séries: Agonista:1 } \\
\text { à 4/Antagonista: } 4 \\
\text { à } 6 \\
\text { Intervalo: } 4 \\
\text { minutos } \\
\text { Intervalo/Exercíci } \\
\text { os:4 minutos } \\
\text { Intensidade: } \\
\text { Agonista: } 6 \text { RM- } \\
\text { 3RM; Antagonista: } \\
\text { 6RM-3RM }\end{array}$ & $\begin{array}{c}\uparrow 1 \mathrm{RM}, \text { força } \\
\text { e velocidade } \\
\text { de pico }\end{array}$ & $6 / 15$ \\
\hline $\begin{array}{l}\text { HAKKINEN et } \\
\text { al., (1998). }\end{array}$ & $\begin{array}{c}21 \\
\text { Homens } \\
40-75 \\
\text { anos } \\
21 \\
\text { Mulheres } \\
36-70 \\
\text { anos } \\
\text { Experiênc } \\
\text { ia não } \\
\text { relatada }\end{array}$ & $\begin{array}{c}\text { Secção } \\
\text { Transversa } \\
\text { do } \\
\text { Quadríceps; } \\
\text { Força } \\
\text { máxima; } \\
\text { Força } \\
\text { explosiva }\end{array}$ & $\begin{array}{c}\text { Agonista: } \\
\text { Quadríceps } \\
\text { Antagonista: } \\
\text { Bíceps } \\
\text { Femoral } \\
\text { Exercícios: Leg } \\
\text { Press/Cadeira } \\
\text { Extensora } \\
\text { Séries: } 3-5 \times 5-15 \\
\text { Intervalo: } \\
\text { Cadência: Final do } \\
\text { período, ações } \\
\text { rápidas } \\
\text { Intensidade: } \\
\text { Agonista: } 50-\end{array}$ & 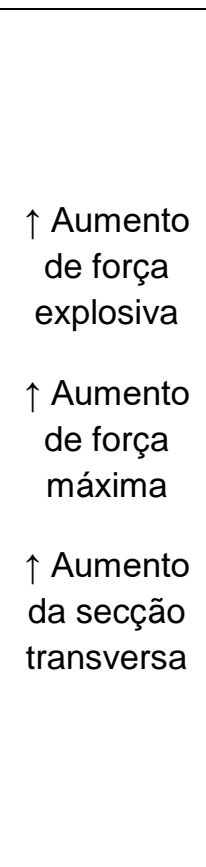 & $6 / 15$ \\
\hline
\end{tabular}


Exercício físico e desempenho muscular

\begin{tabular}{|l|l|l|l|l|l|}
\hline & & & $80 \%$ de 1RM & & \\
& & & & & \\
& & & & & \\
\hline
\end{tabular}

Legenda: RM: Repetição (ões) Máxima(s). DISCUSSÃO

Segundo os dados obtidos no presente estudo, o método agonistaantagonista se mostrou eficaz para as variáveis relacionadas ao desempenho muscular (volume total de trabalho, atividade eletromiográfica e número de repetições) quando comparado ao método tradicional. Tais respostas foram observadas, com a aplicação do MAA, utilizando estímulos para o músculo antagonista por meio de exercícios de força, alongamento estático e facilitação neuromuscular proprioceptiva.

Dois estudos observaram melhora no volume total de trabalho utilizando somente exercício de força (PAZ et al., 2016; ROBBINS et al., 2010). Todavia, no primeiro estudo, a intensidade aplicada para musculatura agonista e antagonista foi de dez repetições máximas (10RM), enquanto no segundo foi utilizado a intensidade quatro repetições máximas (4RM). Paz et al., 2012 por sua vez, analisou a mesma variável (volume total de trabalho), porém, utilizando facilitação neuromuscular proprioceptiva como estímulo à musculatura antagonista, onde seus resultados corroboram com os estudos anteriores. Tais disparidades nas intensidades utilizadas impedem a utilização do método de forma precisa, quando o objetivo for melhora no volume total de trabalho.

Ao analisar os efeitos na atividade EMG durante a utilização do MAA, observouse que a principal intensidade utilizada, ao utilizar apenas o treinamento de força (TF) como estímulo, é de 10 RM (PAZ et al., 2016; PAZ et al., 2014). Quando se optou pelo alongamento estático como estimulo ao

antagonista, foi usada a intensidade de 40 segundos em zona de desconforto do indivíduo (MIRANDA et al., 2015). Ambas as situações se mostraram eficientes quando o objetivo for melhora de ativação EMG. Em contrapartida, a utilização de FNP parece não ser tão eficiente no aumento da atividade EMG durante o MAA (PAZ et al., 2012).

Foram vistos aumentos significativos no número de repetições, tanto nos estudos que utilizaram somente TF, cujas intensidades utilizadas variam entre $90 \%$ de 10 RM e $100 \%$ de 10 RM (MAIA et al., 2014; PAZ et al., 2012; NOBRE, FIGUEIREDO, SIMÃO, 2010), como no estudo de Miranda 2015, que utilizou 40 segundos de alongamento estático em zona de desconforto como estímulo à musculatura antagonista.

\section{CONCLUSÃO}

Apesar das melhorias supracitadas observadas na utilização do MAA, tais achados mostram não ser consensual qual intensidade deve ser aplicada na sua utilização, seja utilizando somente 0 TF, alongamento estático, ou até mesmo a facilitação neuromuscular proprioceptiva (FNP). Tal dificuldade poderá impedir utilização do MAA de forma precisa e objetiva, evitando assim a utilização de intensidades desnecessárias ao sujeito, ou até mesmo sua subestimativa. Dessa forma, são necessários estudos que avaliem qual deve ser a intensidade ideal aplicada a musculatura antagonista, seja em treinamento de força ou em alongamento estático, para otimizar os resultados nas variáveis apresentadas no presente estudo. 


\section{Exercício físico e desempenho muscular}

\section{REFERÊNCIAS}

COSTA, B. D. de V. et al. Acute effects of the intensity in the agonistantagonist paired-set on the neuromuscular performance. Revista brasileira de ciência e movimento, p. 149-156, 2020.

CUNHA, R. et al. Effects of short-term isokinetic training with reciprocal knee extensors agonist and antagonist muscle actions: a controlled and randomized trial. Brazilian Journal of physical therapy, v. 17, p. 137-145, 2013.

HAKKINEN, K. et al. Changes in agonist-antagonist EMG, muscle CSA, and force during strength training in middle-aged and older people. Journal of applied physiology, v. 84, n. 4, p. 1341-1349, 1998.

MAEO, S. et al. Neuromuscular adaptations following 12-week maximal voluntary co-contraction training. European Journal of Applied Physiology, v. 114, n. 4, p. 663-673, 2014.

MAIA, M. F. et al. Effects of different rest intervals between antagonist paired sets on repetition performance and muscle activation. The Journal of Strength \& Conditioning Research, v. 28, n. 9, p. 2529-2535, 2014.

MIRANDA, $\mathrm{H}$. et al. Acute effects of antagonist static stretching in the inter-set rest period on repetition performance and muscle activation. Research in Sports Medicine, v. 23, n. 1, p. 37-50, 2015.

MIRANDA, $\mathrm{H}$. et al. Efeito agudo do alongamento estático nos antagonistas sobre o teste de repetições máximas para os músculos agonistas. Revista Brasileira de Ciência e Movimento, v. 22, n. 2, p. 19-26, 2014

NOBRE, M.; FIGUEIREDO, T.; SIMÃO, R. Influência do método agonista-antagonista no desempenho do treinamento de força para membros inferiores. RBPFEX-Revista Brasileira de Prescrição e Fisiologia do Exercício, v. 4, n. 22, 2010.

PAZ, G. A. et al. Effect of agonist-antagonist method compared to traditional on muscular volume and activation. Revista Brasileira de Atividade Física e Saúde, v. 19, n. 1, p. 54-63, 2014.

PAZ, G. A. et al. Maximal Exercise Performance and Electromyography Responses after Antagonist Neuromuscular Proprioceptive Facilitation: A Pilot Study. Journal of Exercise Physiology Online, v. 15, n. 6, 2012.

PAZ, G. A. et al. Volume load and neuromuscular fatigue during an acute bout of agonist-antagonist paired-set vs. traditional-set training. The Journal of Strength \& Conditioning Research, v. 31, n. 10, p. 2777-2784, 2016.

PAZ, G. et al. Effect of Agonist-Antagonist Paired Set Training vs. Traditional Set Training on PostResistance Exercise Hypotension. Journal of Exercise Physiology Online, v. 17, n. 6, 2015.

ROBBINS, D. W. et al. Effects of agonist-antagonist complex resistance training on upper body strength and power development. Journal of sports sciences, v. 27, n. 14, p. 1617-1625, 2009.

ROBBINS, D. W. et al. Physical performance and electromyographic responses to an acute bout of paired set strength training versus traditional strength training. The Journal of Strength \& Conditioning Research, v. 24, n. 5, p. 1237-1245, 2010.

ROBBINS, D. W. et al. The effect of a complex agonist and antagonist resistance training protocol on volume load, power output, electromyographic responses, and efficiency. The Journal of Strength \& Conditioning Research, v. 24, n. 7, p. 1782-1789, 2010. 


\section{Exercício físico e desempenho muscular}

SMART, N. A. et al. Validation of a new tool for the assessment of study quality and reporting in exercise training studies: TESTEX. JBI Evidence Implementation, v. 13, n. 1, p. 9-18, 2015.

STOCK, M. S.; THOMPSON, B. J. Sex comparisons of strength and coactivation following ten weeks of deadlift training. Journal Musculoskelet Neuronal Interact, v. 14, n. 3, p. 387-97, 2014. 